\title{
Inositol-requiring enzyme 1-mediated endoplasmic reticulum stress triggers apoptosis and fibrosis formation in liver cirrhosis rat models
}

\author{
TIANPENG JIANG，LIZHOU WANG，XING LI，JIE SONG，XIAOPING WU and SHI ZHOU \\ Department of Radiology, Affiliated Hospital of Guiyang Medical College, Guiyang, Guizhou 550004, P.R. China
}

Received February 6, 2014; Accepted September 12, 2014

DOI: $10.3892 / \mathrm{mmr} .2014 .3020$

\begin{abstract}
Long-term and advanced cirrhosis is usually irreversible and often coincides with variceal hemorrhage or development of hepatocellular carcinoma; therefore, liver cirrhosis is a major cause of morbidity and mortality globally. The aim of the present study was to investigate the specific mechanism behind the formation of fibrosis or cirrhosis using rat models of hepatic fibrosis. The cirrhosis model was established by intraperitoneally administering dimethylnitrosamine to the rats. Hematoxylin and eosin staining was performed on the hepatic tissues of the rats to observe the fibrosis or cirrhosis, and western blot analysis was employed to detect $\alpha$-smooth muscle actin and desmin protein expression. Flow cytometric analysis was used to examine early and late apoptosis, and the protein and mRNA expression of endoplasmic reticulum (ER) stress-associated unfolded protein response (UPR) pathway proteins and apoptotic proteins [C/EBP homologous protein (CHOP) and caspase-12] was detected by western blotting and the reverse-transcription polymerase chain reaction, respectively. The results indicated that the cirrhosis model was established successfully and that fibrosis was significantly increased in the cirrhosis model group compared with that in the normal control group. Flow cytometric analysis showed that early and late apoptosis in the cirrhosis model was significantly higher compared with that in the control group. The expression of the UPR pathway protein inositol-requiring enzyme (IRE) 1, as well as the expression of $\mathrm{CHOP}$, was increased significantly in the cirrhotic rat tissues compared with that in the control group tissues $(\mathrm{P}<0.05)$. In conclusion, apoptosis was clearly observed in the hepatic tissue of cirrhotic rats, and the apoptosis was caused by activation of the ER stress-mediated IRE1 and CHOP.
\end{abstract}

Correspondence to: Dr Shi Zhou, Department of Radiology, Affiliated Hospital of Guiyang Medical College, 28 Guiyi Road, Guiyang, Guizhou, 550004, P.R. China

E-mail: zhoushiguiyang@yeah.net

Key words: liver cirrhosis, apoptosis, ER stress, IRE1, CHOP

\section{Introduction}

Liver cirrhosis is a long-term consequence of hepatitis virus infection or hepatic injury caused by alcohol abuse, which is characterized by dysfunction with extensive accumulation of fibrous tissue in the liver (1). Long-term and advanced cirrhosis is usually irreversible and is often associated with variceal hemorrhage or the development of hepatocellular carcinoma; therefore, liver cirrhosis is a major worldwide cause of morbidity and mortality (2). In the liver tissue, quiescent hepatic stellate cells (HSCs) and liver myofibroblasts are the main sources of hepatic extracellular matrix (ECM) and play a key role in the pathogenesis $(3,4)$. Although the cell proliferation and migration of HSCs and portal myofibroblasts are a pathogenic cause of hepatic fibrosis, previous studies $(5,6)$ have indicated that the apoptotic cell death of liver cells may also be involved in the formation of hepatic fibrosis and cirrhosis.

Previous studies have commonly reported that endoplasmic reticulum (ER) stress plays a crucial role in the induction and regulation of apoptosis $(7,8)$. There are numerous pathways involved in ER stress, such as ER-associated protein degradation and the unfolded protein response (UPR) (9) The UPR pathway also activates several proteins, including inositol-requiring enzyme (IRE) 1, protein kinase R-like ER kinase (Perk) and activating transcription factor 6 (ATF6) (10). The above proteins can further activate the apoptosis of the cells.

In the present study, the specific mechanisms for the development of cirrhosis and hepatic fibrosis were investigated in the liver tissue of a rat model of cirrhosis. It was proposed that the elucidation of these mechanisms could lead the way to therapeutic methods for patients with liver cirrhosis.

\section{Materials and methods}

Animal treatment and cirrhosis model establishment. Wistar rats (Animal Center of Guiyang Medical College, Guiyang, China) aged between four and six weeks $(n=60)$ were used in this study. All of the animal experiments were performed according to the US National Institutes of Health 1991 guidelines, and approved by the Ethics Committee of Guiyang Medical College (Guiyang, China). For the establishment of 
A

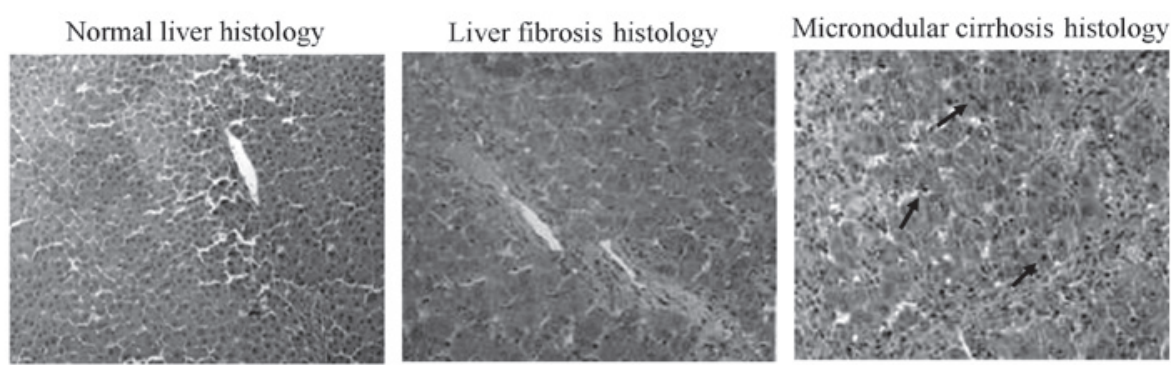

B

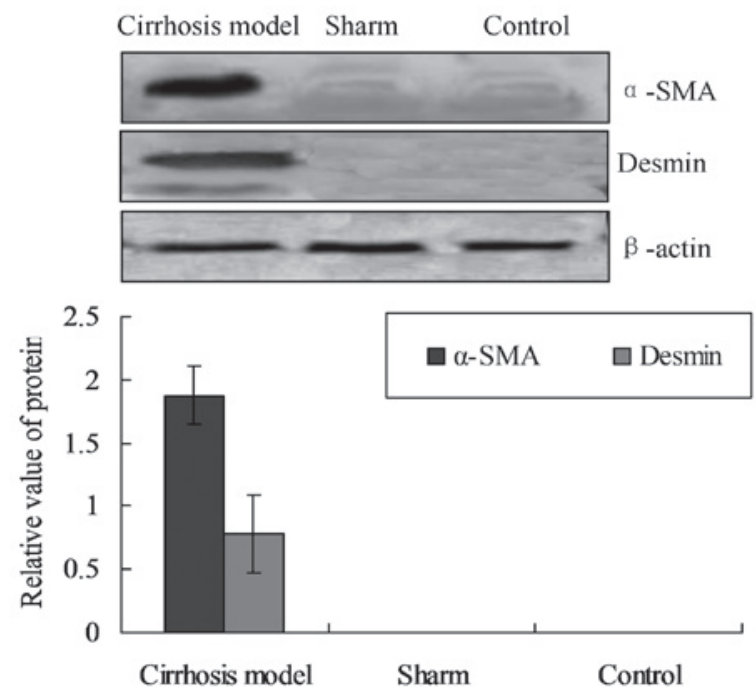

Figure 1. Fibrosis formation in the cirrhosis model, sham and control groups: (A) Photomicrographs of liver sections stained with hematoxylin and eosin from the rats receiving dimethylnitrosamine intraperitoneally. Arrows represent the positive staining cells (magnification, x200). (B) Changes in expression of hepatic $\alpha$-SMA and desmin in the livers of cirrhotic, normal and sham rats. Expression of $\alpha$-SMA and desmin was analyzed using western blotting. SMA, smooth muscle actin. The photomicrographs are all from the rats receiving dimethylnitrosamine. The normal histology image was from the control rats.

experimental hepatic cirrhosis, rats $(n=20)$ were intraperitoneally administered with dimethylnitrosamine (Sigma-Aldrich, St. Louis, MO, USA) dissolved in saline at $10 \mathrm{mg} / \mathrm{kg}$ rat body weight, on three consecutive days a week for five continuous weeks. An equivalent number of rats $(n=20)$ were treated with saline only as a sham group, and 20 untreated rats formed a control group.

Immunohistochemical examination. After the five week treatment period, the rats were anaesthetized with intravenous pentobarbital sodium $(40 \mathrm{mg} / \mathrm{kg})$ and sacrificed by cervical dislocation, and the liver tissues were isolated and fixed with $10 \%$ neutralized formaldehyde or $70 \%$ ethanol and embedded in paraffin. For the immunohistochemical examination of desmin and $\alpha$-smooth muscle actin ( $\alpha$-SMA), the tissue sections were incubated with monoclonal anti-mouse desmin (Santa Cruz Biotechnology, Inc., Santa Cruz, CA, USA) and monoclonal anti-mouse $\alpha$-SMA (Santa Cruz Biotechnology, Inc.) antibodies for $1 \mathrm{~h}$, respectively. The immunohistochemical procedures were performed according to the method described by Xu et al (11).

Hematoxylin and eosin (HE) staining. 10 weeks after the start of the experiment the animals were anaesthetized with $40 \mathrm{mg} / \mathrm{kg}$ intravenous pentobarbital sodium and sacrificed and the livers were recovered for histological analysis. The analysis was performed by an experienced pathologist who was unaware of the experimental groups. Liver specimens were fixed in buffered $10 \%$ formalin, embedded in paraffin and stained with $\mathrm{HE}$ and picrosirius red to evaluate the extent of liver fibrosis. A microscope (2124002; Sigma-Aldrich) was used to evaluate the slides. The classification of the tissue slides was in accordance with the methods of Goldani et al (12).

RNA extraction and semi-quantitative reverse transcription-polymerase chain reaction $(R T-P C R)$. To measure the transcriptional levels of the ER-stress associated agents, semi-quantitative RT-PCR assays were designed, which included IRE1, cleaved ATF6 and p-Perk. $\beta$-actin served as the internal control. Total RNA $(10 \mu \mathrm{g})$ was obtained using the RNAsimple Total RNA Kit [Tiagen Biotech (Beijing) Co., Ltd., China]. Reverse transcription was performed using the SuperScript ${ }^{\mathrm{TM}}$ III First-Strand Synthesis System (Invitrogen Life Technologies, Carlsbad, CA USA). Briefly, $2 \mu \mathrm{g}$ total RNA, $200 \mathrm{U}$ MMLV reverse transcriptase and $50 \mathrm{pM}$ oligo (dT20) were mixed in a volume of $20 \mu \mathrm{l}$. The mixture was incubated at $50^{\circ} \mathrm{C}$ for $50 \mathrm{~min}$ and inactivated by heating at $85^{\circ} \mathrm{C}$ for $5 \mathrm{~min}$. To remove the RNA, $1 \mu \mathrm{l}$ Rase $\mathrm{H}$ was added to the mixture, followed by incubation at $37^{\circ} \mathrm{C}$ for $20 \mathrm{~min}$. Aliquots $(2 \mu \mathrm{l})$ of the $\mathrm{RT}$ reaction products were amplified by PCR in a volume of $50 \mu \mathrm{l}$ under the following conditions: $94^{\circ} \mathrm{C}$ for $30 \mathrm{sec}, 54^{\circ} \mathrm{C}$ for $30 \mathrm{sec}$ and $72^{\circ} \mathrm{C}$ for $40 \mathrm{sec}$. Next, the products were electrophoresed on $1.4 \%$ agarose gels. Images of each PCR product were then captured using a CCD camera (Nikon Coporation, Tokyo, Japan) and analyzed using NIH Imager beta version 2 (Bio-Rad Laborotories, 
A
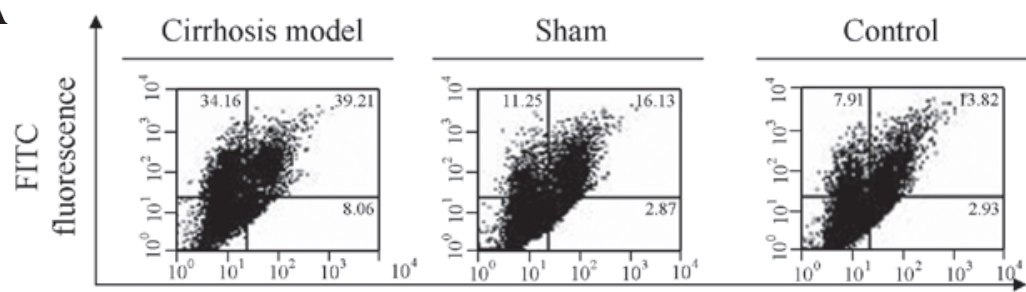

$\mathbf{B}$

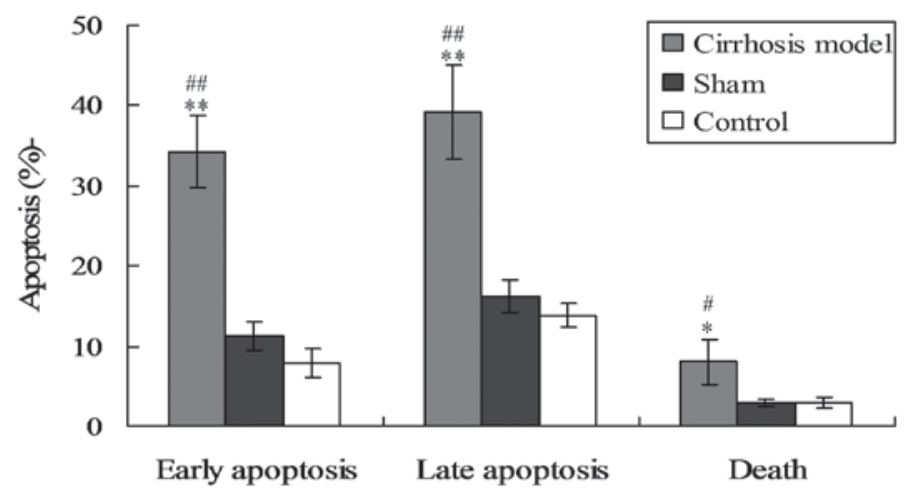

Figure 2. Apoptosis is observed in the cirrhosis model. (A) Annexin V/PI double staining assays of cells in three groups. The X axis indicates the number of PI-stained cells; the Y axis indicates the number of Annexin V-FITC-stained cells. The percentages of the cells labeled with only Annexin V (counted in Q1 region) in each preparation are shown in each figure. (B) Statistical analysis. Results for three independent experiments are shown. The normalized data against the sham and control are presented as mean \pm standard deviation; ${ }^{* *} \mathrm{P}<0.01$ and ${ }^{*} \mathrm{P}<0.05$ vs. the sham group; ${ }^{\# \prime} \mathrm{P}<0.01$ and ${ }^{\#} \mathrm{P}<0.05$ vs. the control group. PI, propidium iodide; FITC, fluorescein isothiocyanate.

A

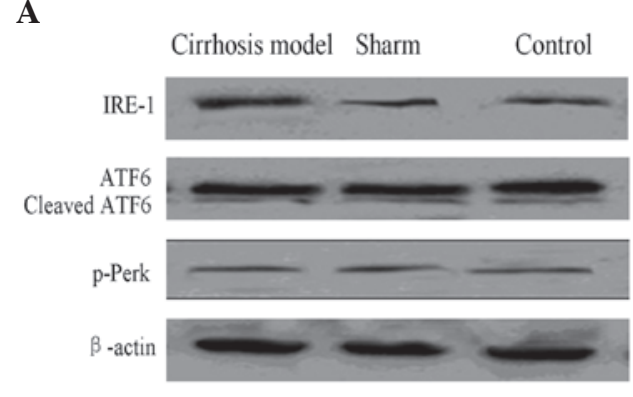

C

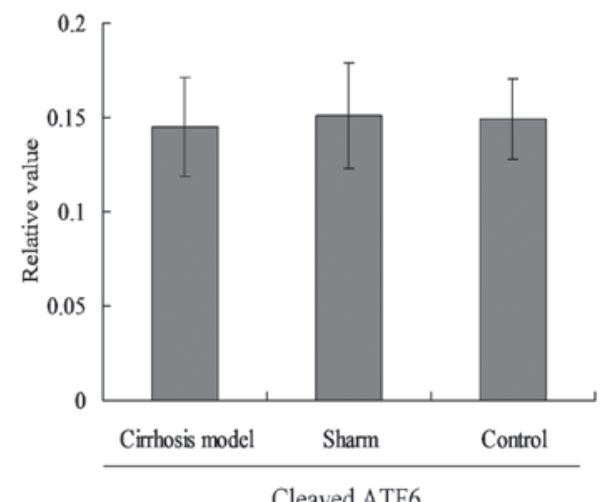

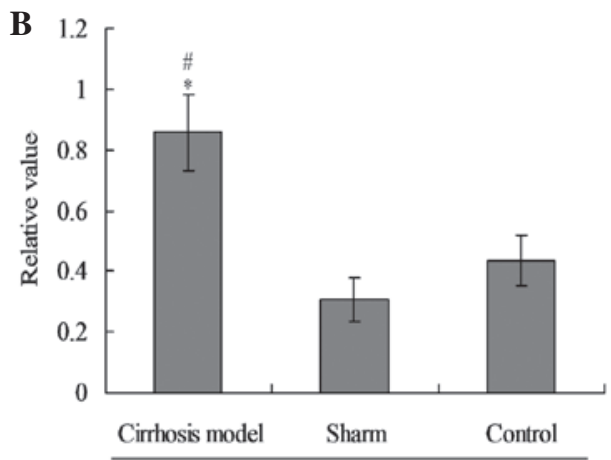

D

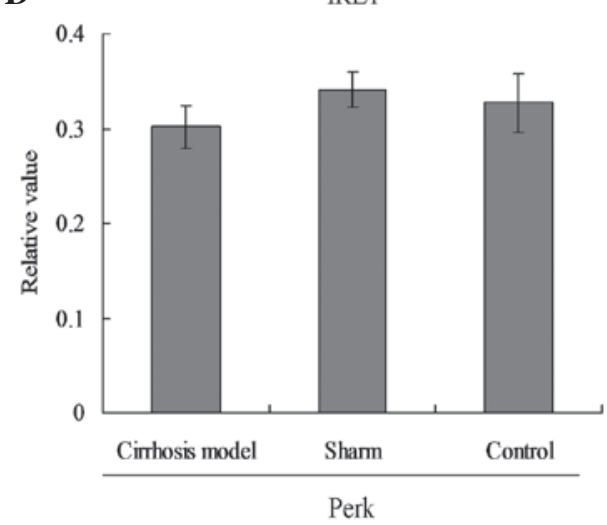

Figure 3. Examination of unfolded protein response pathway proteins. (A) Western blot analysis of IRE1, p-Perk and cleaved ATF6 proteins. (B-D) Statistical analysis of (B) IRE1, (C) cleaved ATF6 and (D) p-Perk protein. " $\mathrm{P}<0.05$ vs. the sham group; ${ }^{\text {}} \mathrm{P}<0.05$ vs. the control group. IRE, inositol-requiring enzyme; p-Perk, phosphorylated-protein kinase R-like endoplasmic reticulum kinase; ATF, activating transcription factor.

Inc., Hercules, CA, USA). Relative transcriptional values are presented as a ratio of the signal value of the specific PCR product compared with individual $\beta$-actin.
Western blot analysis. In order to examine the protein expression using western blot analysis, the cells were lysed in the sample buffer for SDS-PAGE. The cell lysate was subjected 
A

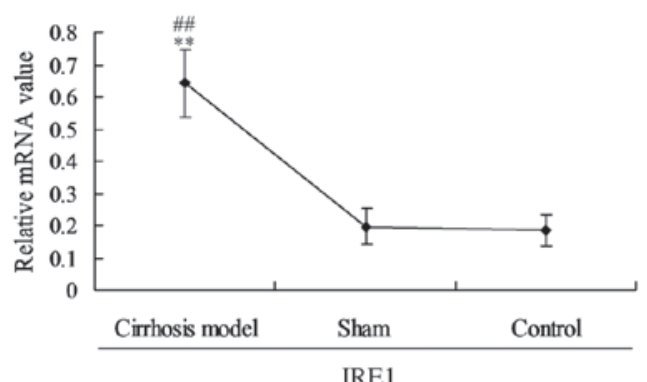

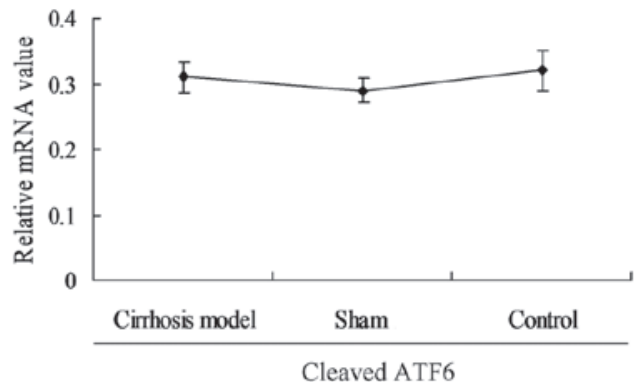

C

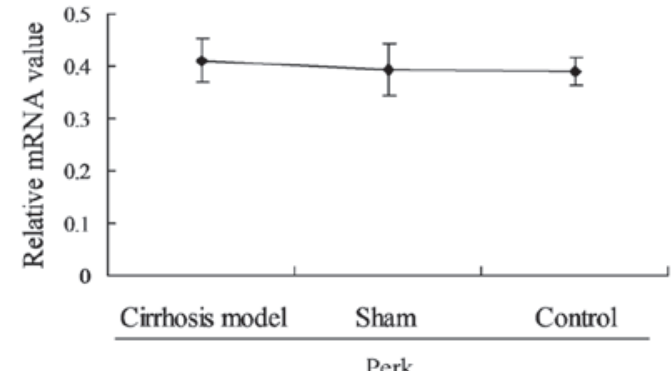

Figure 4. Examination of mRNA levels of ER stress (unfolded protein response)-associated genes. Semi-quantitative analysis of (A) IRE1, (B) cleaved ATF6 and (C) Perk mRNA. ${ }^{* *} \mathrm{P}<0.01$ vs. the sham group; ${ }^{\# \#} \mathrm{P}<0.01$ vs. the control group. ER, endoplasmic reticulum; IRE, inositol-requiring enzyme; ATF, activating transcription factor; Perk, protein kinase R-like ER kinase.

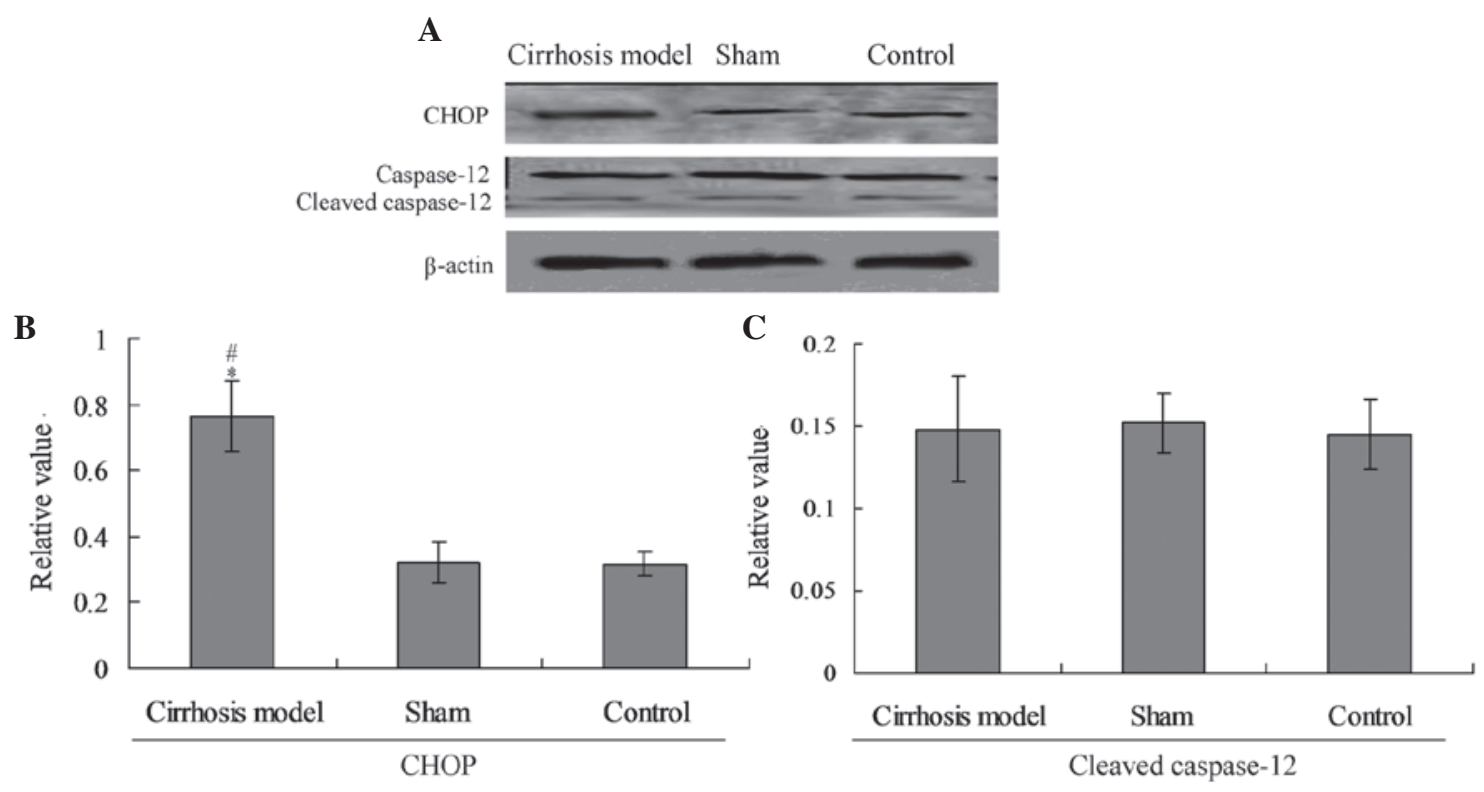

Figure 5. Apoptosis-related protein expression. (A) Levels of CHOP and cleaved caspase-12 protein were evaluated by western blot analysis. (B and C) Statistical analysis was performed for (B) CHOP and (C) cleaved caspase-12 protein. ${ }^{~} \mathrm{P}<0.05$ vs. the sham group; ${ }^{~} \mathrm{P}<0.05$ vs. the control group. CHOP, C/EBP homologous protein.

to SDS-PAGE and proteins were electroblotted onto polyvinylidene difluoride membranes. Subsequent to blocking with phosphate-buffered saline containing 5\% skimmed milk, the membrane was incubated with abbit anti-rat IRE1 monoclonal antibody (Santa Cruz Biotechnology, Inc.), rabbit anti-rat cleaved ATF6 polyclonal antibody (Santa Cruz Biotechnology, Inc.), rabbit anti-rat cleaved caspase-12 monoclonal antibody (Santa Cruz Biotechnology, Inc.) and rabbit anti-rat CHOP monoclonal antibody (Santa Cruz Biotechnology, Inc.). Immunoreactive proteins were visualized using an enhanced chemiluminescence reagent (Invitrogen Life Technologies).
Flow cytometry assay. Cell viability was assessed by flow cytometry, which analyzed Annexin V-fluorescein isothiocyanate (FITC) binding and propidium iodide (PI; Trevigen, Inc., Gaithersburg, MD, USA) uptake simultaneously. Briefly, cells were harvested and resuspended in Annexin V-FITC binding buffer (Invitrogen Life Technologies) and incubated with Annexin V-FITC (Invitrogen Life Technologies, CA, USA) and PI (5lg ml-1) (Invitrogen Life Technologies) in the dark at room temperature for $15 \mathrm{~min}$. Samples were then analyzed using a FACScan flow cytometer (Becton-Dickinson, Oxford, UK). Fluorescence was measured using a 530/30 
band filter (FL-1) to monitor Annexin V-FITC binding and a 585/42 band filter (FL-2) to monitor PI uptake.

Statistical analysis. Data are presented as the mean \pm standard deviation. Statistical analyses were performed using the unpaired Student's t-test or analysis of variance. Differences were considered to be statistically significant at $\mathrm{P}<0.05$.

\section{Results}

Increased fibrosis in the cirrhosis rat model. The analysis was performed in the 40 cirrhosis rats $(20$ rats in the treated group and sham group, respectively) and 20 normal rats that survived through five weeks of the treatment period by the time of sacrifice. Cirrhosis was significantly more prevalent in the cirrhosis group than in the sham and control groups $(\mathrm{P}<0.01)$; however, no significant difference was observed between the sham and control groups $(\mathrm{P}>0.05)$. The histology of the normal liver and liver tissues exhibiting fibrosis and cirrhosis is shown in Fig. 1A. The liver tissues were isolated and subjected to western blot analysis in a consistent manner. The results indicated that hepatic expression of $\alpha$-SMA and desmin in the cirrhosis group was increased compared with that in the sham and control groups (Fig. 1B). These findings strongly suggested that the cirrhosis model was established successfully.

Induction of hepatic cell apoptosis in cirrhotic rats. In the present study, it was hypothesized that cirrhosis may be caused by the apoptosis of hepatic cells. Thus, the apoptosis of the hepatic tissues was observed in each group. Flow cytometric analysis showed that the levels of early apoptosis, late apoptosis and cell death in the cirrhosis model were significantly higher compared with those in the sham and control groups (Fig. 2) $(\mathrm{P}<0.01$ for early and late apoptosis, $\mathrm{P}<0.05$ for cell death).

ER stress UPR pathways are involved in cirrhosis-mediated apoptosis. In the present study, three main UPR factors of ER stress, IRE1, Perk and ATF6, were analyzed by western blot assay (Fig. 3) and the RT-PCR (Fig. 4). The results showed that the expression of IRE1 in the cirrhosis group was increased significantly compared with that in the sham and control groups (Fig. 3A and $\mathrm{B}, \mathrm{P}<0.05$ ); however, no evident changes were observed in cleaved ATF6 and phosphorylated (p)-Perk protein among the three groups (Fig. 3A, C and D).

The expression of mRNA for the above UPR proteins was analyzed with semi-quantitative PCR. As indicated in Fig. 4, when compared with the sham and control groups, the mRNA levels of IRE1 were increased significantly in the cirrhosis group (Fig. 4A, $\mathrm{P}<0.01$ ). Notably, no significant differences were observed in cleaved ATF6 and Perk levels among the three groups (Fig. 4B and C).

C/EBP homologous protein $(\mathrm{CHOP})$ changes mediate the ER stress-associated apoptosis in cirrhotic rats. To explore the specific pathway causing the apoptosis in hepatic tissues, the cellular protein levels of cleaved caspase-12 and CHOP were evaluated by individual western blot analysis (Fig. 5A). The results indicated that the expression of CHOP in the cirrhosis model was significantly increased compared with that in the control group $(\mathrm{P}<0.05)$. No significant differences were observed in cleaved caspase-12 levels among the three groups (Fig. 5B).

\section{Discussion}

Activated HSCs and portal myofibroblasts act as major cellular sources of ECM deposition in liver fibrosis/cirrhosis following hepatic injury and/or inflammation. Conversely, during spontaneous recovery from liver fibrosis, activated HSCs and portal myofibroblasts undergo apoptotic cell death (13). Thus, the apoptosis of liver cells and portal myofibroblasts is closely involved in recovery from liver fibrosis/cirrhosis. Although a few studies have explored the association between cirrhosis and apoptosis $(14,15)$, the present study is the first, to the best of our knowledge, that investigates the specific mechanism of cirrhosis-mediated apoptosis in liver tissues.

In the present study, fibrosis formation was observed in the cirrhosis rat model. The results indicated that the fibrosis was significantly increased in the cirrhosis model group compared with that in the normal control group. It has been shown that, for fibrosis or cirrhosis formation, cells must express fibrosisrelated proteins (16); thus, $\alpha$-SMA and desmin proteins were detected in the hepatic tissues in the present study. The marked increase in fibrous tissue expansion and hepatic expression of $\alpha$-SMA and desmin suggested that the formation of the fibrosis or cirrhosis was caused by the $\alpha$-SMA and desmin expression. We speculated that the potential mechanism for the enhanced level of $\alpha$-SMA and desmin may have been due to the apoptosis of hepatic cells.

In order to investigate the specific mechanism underlying the cirrhosis-related apoptosis, the ER stress (UPR pathway)-associated protein levels, including p-Perk, IRE1 and cleaved ATF6, were detected (17). It was found that IRE1 protein and mRNA were activated in the hepatic tissue of the cirrhosis model; therefore, the IRE1 UPR pathway may be involved in cirrhosis-related apoptosis. The results of cirrhosis-related apoptosis may assist in examining the role of fibrosis in the progression of liver cirrhosis. A previous study showed that the activation of IRE1 could activate the ER-associated apoptosis and cell death (18). The data strongly indicated the emergence of ER stress for the cirrhosis rat model; therefore, we propose that ER stress may participate in the pathogenic process of fibrosis formation in hepatic cirrhosis.

In a study by Zhao et al (19), ER stress-associated factors (cleaved caspase-12 and CHOP) were detected to help identify the specific apoptotic factors involved in the pathogenesis of cirrhosis. The study reavealed that simvastatin protects MG63 cells from oxidative stress-induced apoptosis through downregulation of caspase-3, caspase- 9 and caspase-12 activation. A number of studies have found that cleaved caspase-12 can activate caspase-3 and trigger apoptosis and that $\mathrm{CHOP}$ can directly induce ER stress-associated apoptosis $(20,21)$. In the present study, no significant changes were observed in the cleavage of caspase-12 protein (activated) among the groups ( $\mathrm{P}>0.05)$; however, the level of $\mathrm{CHOP}$ in the cirrhotic rats was significantly increased compared with that in the sham and control groups. From these results, it was deduced that the ER stress-associated protein CHOP was activated, which 
subsequently induced the apoptosis. This indicated that the fibrosis was caused by CHOP activation induced by ER stress.

In conclusion, the protein expression of the UPR pathway protein, inositol-requiring enzyme (IRE) 1, and the expression of CHOP was increased significantly in the cirrhotic rat tissues. The evident apoptosis observed in the hepatic tissue of cirrhotic rats, which was caused by the activation of the ER stress-mediated IRE1 and CHOP.

\section{References}

1. Zhang C, Wang Y, Chen H, Yang G, Wang S, Jiang M, Cong L, Yuan L, Li H and Jia Y: Protective effect of the herbal medicine Gan-fu-kang against carbon tetrachloride-induced liver fibrosis in rats. Mol Med Rep 8: 954-962, 2013.

2. Kim WH, Matsumoto K, Bessho K and Nakamura T: Growth inhibition and apoptosis in liver myofibroblasts promoted by hepatocyte growth factor leads to resolution from liver cirrhosis. Am J Pathol 166: 1017-1028, 2005.

3. Alcolado R, Arther MJP and Iredale JP: Pathogenesis of liver fibrosis. Clin Sci (Lond) 92: 103-112, 1997.

4. Yoshiji H, Noguchi R, Ikenaka Y, Kaji K, Aihara Y, Douhara A Yamao J, Toyohara M, Mitoro A, Sawai M, Yoshida M, Morioka C, Fujimoto M, Uemura M and Fukui H: Combination of branched-chain amino acid and angiotensin-converting enzyme inhibitor improves liver fibrosis progression in patients with cirrhosis. Mol Med Rep 5: 539-544, 2012.

5. Ramadori G and Saile B: Mesenchymal cells in the liver - one cell type or two? Liver 22: 283-294, 2002.

6. Cha JH, Bae SH, Kim HL, Park NR, Choi ES, Jung ES, Choi JY and Yoon SK: Branched-chain amino acids ameliorate fibrosis and suppress tumor growth in a rat model of hepatocellular carcinoma with liver cirrhosis. PLoS One 8: e77899, 2013.

7. Yung HW, Korolchuk S, Tolkovsdy AM, Charnock-Jones DS and Burton GJ: Endoplasmic reticulum stress exacerbates ischemia-reperfusion-induced apoptosis through attenuation of Akt protein synthesis in human choriocarcinoma cells. FASEB J 21: 872-884, 2007

8. Moenner M, Pluquet O, Bouchecareilh M and Chevet E: Integrated endoplasmic reticulum stress responses in cancer. Cancer Res 67: 10631-10634, 2007.

9. Banjerdpongchai R, Punyati P, Nakrob A, Pompimon W and Kongtawelert P: 4'-Hydroxycinnamaldehyde from Alpinia galangal (Linn.) induces human leukemic cell apoptosis via mitochondrial and endoplasmic reticulum stress pathways. Asian Pac J Cancer Prev 12: 593-598, 2011.
10. Hung JY, Hsu YL, Ni WC, Tsai YM, Yang CJ, Kuo PL and Huang MS: Oxidative and endoplasmic reticulum stress signaling are involved in dehydrocostuslactone-mediated apoptosis in human non-small cell lung cancer cells. Lung Cancer 68: 355-365, 2010.

11. Xu K, Liu XN, Zhang HB, An N, Wang Y, Zhang ZC and Wang YN: Replication-defective HSV-1 effectively targets trigeminal ganglion and inhibits viral pathopoiesis by mediating interferon gamma expression in SH-SY5Y cells. J Mol Neurosci 53: 78-86, 2014.

12. Goldani HA, Matte US, Ramos AR, Costa TG, Winkelmann LV, Meureer L, Vieira SM, Kieling CO and Silveira TR: The role of food restriction on $\mathrm{CCl}_{4}$-induced cirrhosis model in rats. Exp Toxicol Pathol 58: 331-337, 2007.

13. Saile B, Matthes N, Neubauer K, Eisenbach C, El-Armouche H, Dudas J and Ramadori G: Rat liver myofibroblasts and hepatic stellate cells differ in CD95-mediated apoptosis and response to TNF-alpha. Am J Physiol Gastrointest Liver Physiol 283: G435-G444, 2002

14. Foster CR, Daniel LL, Daniels CR, Dalal S, Singh M and Singh K: Deficiency of ataxia telangiectasia mutated kinase modulates cardiac remodeling following myocardial infarction: involvement in fibrosis and apoptosis. PLoS One 8: e83513, 2013.

15. Puche JE, Saiman Y and Friedman SL: Hepatic stellate cells and liver fibrosis. Compr Physiol 3: 1473-1492, 2013.

16. Sheng L, Jiao B, Shao L, Bi S, Cheng C, Zhang J and Jiang Y: Probucol inhibits hydrogen peroxide to induce apoptosis of vascular smooth muscle cells. Mol Med Rep 7: 1185-1190, 2013.

17. Xiao B, Han F, Wang HT and Shi YX: Single-prolonged stress induces increased phosphorylation of extracellular signal-regulated kinase in a rat model of post-traumatic stress disorder. Mol Med Rep 4: 445-449, 2011.

18. Lee JH, Kwon EJ and Kim DH: Calumenin has a role in the alleviation of ER stress in neonatal rat cardiomyocytes. Biochem Biophy Res Commun 439: 327-332, 2013.

19. Zhao XH, Xu ZR, Zhang Q and Yang YM: Simvastatin protects human osteosarcoma cells from oxidative stress-induced apoptosis through mitochondrial-mediated signaling. Mol Med Rep 5: 483-488, 2012.

20. Wang X, Shi Q, Xu K, Gao C, Chen C, Li XL, Wang GR, Tian C, Han J and Dong XP: Familial CJD associated PrP mutants within transmembrane region induced $\mathrm{Ctm}$-PrP retention in ER and triggered apoptosis by ER stress in SH-SY5Y cells. PLoS One 6: e14602, 2011.

21. Xu K, Wang X, Shi Q, Chen C, Tian C, Li XL, Zhou RM, Chu YL and Dong XP: Human prion protein mutants with deleted and inserted octarepeats undergo different pathways to trigger cell apoptosis. J Mol Neurosci 43: 225-234, 2011. 